\title{
Impresa, territorio, competitività: riflessioni e prospettive di ricerca
}

\author{
AMEDEO MAIZZA*
}

\begin{abstract}
Obiettivo del paper: Il paper intende presentare le diverse opportunità di ricerca e l'importanza che gli studi manageriali hanno nel rapporto "impresa-territorio".

Metodologia: Review della letteratura.

Risultati: Il territorio può considerarsi il campo da gioco in cui si realizza la sfida competitiva e si forma l'innovazione. Da qui l'esigenza di: i) riposizionare correttamente il ruolo del "locale" nel nuovo contesto competitivo, evitando tuttavia inutili enfatizzazioni, derivanti da sterili campalinismi; ii) sviluppare forme di governance capaci di sintetizzare le diverse specificità territoriali; iii) affrontare l'argomento attraverso metodi più vicini alla tradizione scientifica italiana quali l'approccio sistemico vitale.

Limiti della ricerca: Il lavoro, avendo la finalità di inquadrare il tema del XXIV Convegno della rivista, affronta l'argomento con metodo induttivo, considerando solo talune evidenze empiriche.

Implicazioni pratiche: Quando un territorio riesce a comporre armonicamente la propria offerta, quale sintesi dei suoi prodotti tangibili e intangibili, si generano economie locali che trovano il loro vantaggio competitivo nell'interdipendenza, la complementarietà e la non replicabilità delle loro formule. Esse, infatti, sono in grado di limitare gli effetti omogenizzanti della globalizzazione, mediante il raggiungimento di condizioni del binomio "territorio - prodotto" tali da determinare l'unicità e la notorietà dell'offerta, consentendo il perseguimento di forme di sviluppo sostenibile.

Originalità del lavoro: Il lavoro cerca di sistematizzare i diversi filoni di ricerca sul tema, individuando ambiti e metodi per l'approfondimento degli studi di management. Nel testo, si fa uso di immagini al fine di lasciare al lettore l'interpretazione e le conseguenti riflessioni sul tema.
\end{abstract}

Parole chiave: impresa; territorio; glocalizzazione; prodotto; Approccio Sistemico Vitale

Purpose of the paper: The aim of this paper is to present different research opportunities and to highlight the importance that managerial studies have with regard to the relationship between a company and its geographical area.

Methodology: Literature review.

Findings: Territory can be considered as the field where the competitive challenge takes place, as well as the ground of innovation. Hence, the need to: $i)$ re-consider the correct role of "local", within the new competitive context, though avoiding an useless overemphasis,

Ordinario di Economia e Gestione delle Imprese - Università degli Studi del Salento

e-mail: amedeo.maizza@unisalento.it

sinergie, rivista di studi e ricerche

n. 90 , Gennaio-Aprile 2013, pp. 11-21

ISSN 0393-5108 - DOI 10.7433/s90.2013.03 
deriving from barren parochialism; ii) develop types of governance which would be able to summarize different kinds of local peculiarities; iii) study the relationship between the Firm and the Territory in new ways, closer to Italian scientific tradition, like the Viable System Approach.

Research limitations: As introduction to XXIV Sinergie Annual Simposium, the paper frame the topic through an indictive method, taking account of some empirical consequences.

Practical implications: If a territory harmoniously composes its offer as a synthesis of its tangible and intangible products, local economies will find their competitive advantage into being interdependent, complementary and unrepleaceble. In fact, they can limit the homogenizer effect of globalization, through the development of a "land - brand" duo which makes offer unique and well know, allowing sustainable development.

Originality: The paper attempts to systematize different researches on the topic, opening new pathways in management studies. Images are used, in order to give the reader the possibility to understand and interpreter their suggestions.

Key words: firm; territory; glocalization; product; Viable System Approach

Quando - un anno fa - è stata confermata la scelta di questa sede per l'annuale convegno di Sinergie, ci siamo da subito interrogati sul tema da proporre. Istintivamente, per via degli interessi di studio prevalenti che impegnano il nostro gruppo di lavoro, abbiamo pensato di affrontare la complessa e, solo in parte, nota relazione "impresa-territorio".

$\mathrm{Ci}$ si è però chiesti se tale argomento potesse avere i connotati necessari per un convegno la cui rilevanza scientifica ed il tradizionale appeal sono ben noti a tutti. Il tema, infatti, rappresenta un classico negli studi sullo sviluppo locale, nell'ambito del quale le teorie manageriali non hanno sinora saputo ricavarsi uno spazio continuativo (si pensi però ai fondamentali lavori di Panati, 1980; Lorenzoni, 1990; Varaldo, 1995; Rullani, 1999).

Nel riflettere su questo aspetto, abbiamo anche ricordato alcuni precedenti convegni di Sinergie i quali, con prospettive diverse, avevano già trattato il tema.

Alla luce di queste iniziali perplessità e, quindi, al fine di individuare ambiti di indagine interessanti, si è reso necessario verificare come la predetta relazione "impresa-territorio" sia oggetto di analisi e ricerche nell'ambito dei nostri studi.

Abbiamo così effettuato una rassegna degli articoli comparsi sulle riviste presenti nel database Liuc Essper. Dalla ricerca, condotta indagando i titoli dei paper con le parole chiave "impresa-territorio" (e similari) per i periodici pubblicati dal 2002 al 2012, è emerso che 50 riviste, riferibili a 15 diversi ambiti scientifici, si sono interessate all'argomento in esame, con una produzione di 82 articoli. Da una più dettagliata analisi si evince come il maggior numero di lavori sia stato redatto da autori di riviste di economia, gestione e contabilità. Fra tutte, emerge la rivista Sinergie per numerosità di approfondimenti del rapporto impresa-territorio che è stato trattato prevalentemente nel 2006, ma poi anche nel 2011 (cfr. Tab. 1). 
Tab. 1: II rapporto Impresa - Territorio: literature review

\begin{tabular}{|c|c|c|c|}
\hline \multicolumn{2}{|r|}{ Ambiti scientifici } & \multirow{2}{*}{$\begin{array}{c}\text { Riviste interessate } \\
50\end{array}$} & \multirow{2}{*}{$\begin{array}{c}\text { Articoli pertinenti } \\
82\end{array}$} \\
\hline & 15 & & \\
\hline 1 & Economia, gestione e contabilità & 23 & 48 \\
\hline & di cui: Sinergie & & 11 \\
\hline 2 & Sociologia e problemi sociali & 5 & 5 \\
\hline 3 & Diritto dell'economia e/o bancario & 2 & 5 \\
\hline 4 & Banche e finanza & 4 & 4 \\
\hline 5 & Statistica & 2 & 4 \\
\hline 6 & Politica e filosofia & 3 & 3 \\
\hline 7 & Diritto tributario & 1 & 3 \\
\hline 8 & Economia del lavoro & 2 & 2 \\
\hline 9 & $\begin{array}{l}\text { Economia delle risorse naturali e } \\
\text { ambientali }\end{array}$ & 2 & 2 \\
\hline 10 & Diritto internazionale & 2 & 2 \\
\hline 11 & Commercio, trasporti e turismo & 1 & 1 \\
\hline 12 & Storia & 1 & 1 \\
\hline 13 & Diritto pubblico & 1 & 1 \\
\hline 14 & Diritto amministrativo & 1 & 1 \\
\hline
\end{tabular}

Fonte: elaborazione dell'autore

L'argomento è stato affrontato da Sinergie prevalentemente nel 2006 (cfr. Tab. 2), con 20 articoli, pari al $24,4 \%$ della produzione rilevata nel periodo di riferimento indagato (nel 2005 si è tenuto il convegno Sinergie a Brescia "Impresa e territorio tra pubblico e privato"), seguito dal 2011, con 12 articoli (pari al 14,6\%), e dal 2004 $(11 \%)$.

Pur non avendo la pretesa dell'esaustività, si può asserire che, negli studi manageriali, la relazione impresa-territorio viene prevalentemente affrontata prestando attenzione:

- agli aspetti economici, quindi al make or buy, al radicamento e alla delocalizzazione,

- alle potenzialità paesaggistiche,

- alle risorse naturali,

- alla propensione e agli effetti delle formule sistemiche,

- alla possibile attrattività turistica e degli investimenti,

- all'analisi strategica dei rapporti impresa-stakeholder,

- alla definizione di ambiente dell'impresa. 
Tab. 2: Il rapporto Impresa - Territorio su Sinergie

\begin{tabular}{|cr|}
\hline \multicolumn{2}{|c|}{$\begin{array}{c}\text { Incidenza \% degli articoli } \\
\text { su base annua }\end{array}$} \\
\hline 2002 & $9,8 \%$ \\
2003 & $4,9 \%$ \\
2004 & $11,0 \%$ \\
2005 & $7,3 \%$ \\
2006 & $24,4 \%$ \\
2007 & $9,8 \%$ \\
2008 & $8,5 \%$ \\
2009 & $2,4 \%$ \\
2010 & $7,3 \%$ \\
2011 & $14,6 \%$ \\
\hline
\end{tabular}

Fonte: elaborazione dell'autore

Molto interessanti e numericamente prevalenti appaiono i contributi riguardanti il place marketing ed il tourism marketing management i quali, congiuntamente agli studi sui distretti industriali, hanno contribuito decisamente a far affiorare l'importanza del "contesto" per lo sviluppo reciproco dell'impresa e del territorio su cui essa si insedia.

In quest'attività di ricerca, ho avuto piacere di rileggere anche l'editoriale di Sinergie n. 84, 2011 dei Proff. Baccarani e Golinelli dal titolo "Per una rivisitazione delle relazioni tra impresa e territorio". In estrema sintesi, il messaggio che il saggio riesce egregiamente a trasmettere è il seguente: Il territorio non può essere considerato come un puro e semplice "oggetto", un terreno di conquista, una risorsa da sfruttare. Il territorio deve, invece, intendersi come il campo di gioco in cui si realizza la sfida competitiva, è il luogo ove l'innovazione si forma, o non si forma, in relazione al suo caratterizzarsi per un'atmosfera creativa.

Muovendo, dunque, dalla constatazione dell'interesse crescente del tema per gli studi di management, non si è potuto omettere di rilevare l'attualità dell'argomento che, anche per i noti effetti della crisi economica, è, quasi quotidianamente, oggetto di dibattito.

Si è così proceduto a una rassegna degli articoli comparsi sulle principali testate giornalistiche nazionali (Corriere della Sera, La Repubblica, Il Sole 24 ore, Italia Oggi e Gazzetta del Mezzogiorno) negli ultimi sei mesi, allo scopo di individuare le parole che, più frequentemente, vengono abbinate al tema del "territorio" (fig. 1). 
Fig. 1: Territorio e...

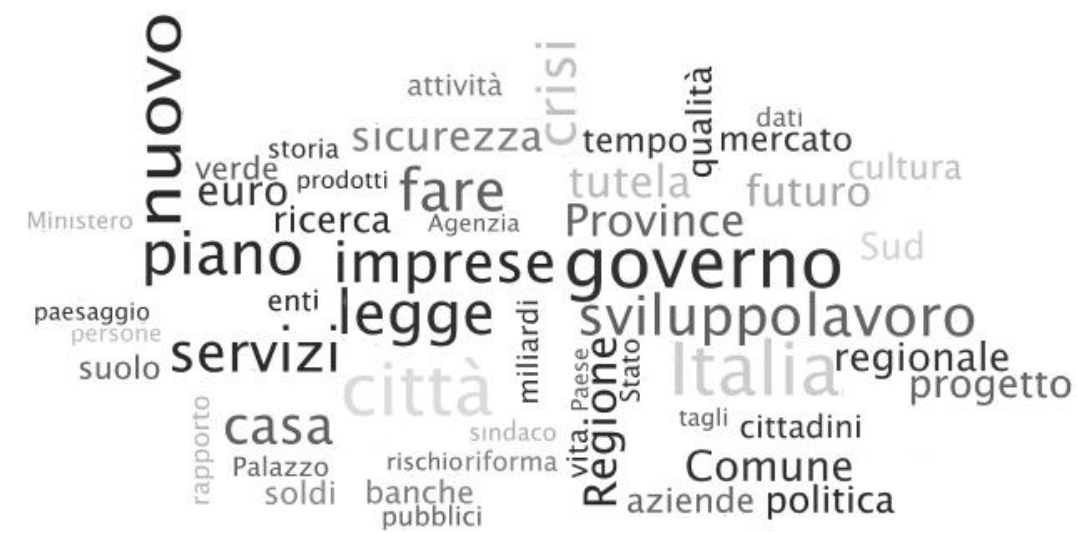

Fonte: elaborazione dell'autore

Da questa analisi, emerge chiaramente come il "territorio" sia protagonista della cronaca, non solo perché al centro dei fatti del giorno di maggior interesse, ma anche perché parlare di "territorio" vuol dire interrogarsi, in un'ottica più specificatamente politica, del suo "governo" e della sua "valorizzazione" a beneficio dell'intera collettività. Molto forte, analizzando i giornali, è l'istanza di una nuova progettualità, che lo tuteli non solo dal punto di vista ambientale, ma anche culturale e che, parallelamente, lo trasformi in volano per superare, in un'ottica di sostenibilità, l'attuale contesto di crisi. Un'esigenza, questa, confermata anche nel momento in cui si passano a considerare le persone e le imprese che, più frequentemente, attirano l'attenzione dei giornali quando si discute di "territorio".

Alla luce di queste brevi considerazioni, emerge, dunque, la necessità di un maggiore impegno degli studiosi di management sul tema che dovrebbe essere affrontato con un approccio di analisi non necessariamente in linea con le principali tendenze in atto in altri contesti ed ambiti geografici, ma attraverso l'adozione di metodi più vicini alla tradizione scientifico - manageriale italiana. Penso in particolare all'approccio sistemico per le ragioni esposte nel prosieguo.

Eccoci dunque ad affrontare il tema "Il territorio come giacimento di vitalità per l'impresa". Perché questo titolo?

La risposta è in parte data dal call for paper, in particolare, nel passaggio iniziale in cui si legge:

"Il territorio è sede della memoria culturale delle generazioni che l'hanno attraversato, vissuto, costruito, a volte distrutto, poi ricostruito e comunque modificato. Il territorio è un patrimonio di storie, unico e irripetibile. Il territorio è come un forziere di valori, conoscenze, cultura, arte, spesso invisibili, perché sepolti sotto una spessa coltre di polvere, prodotta dalla velocità imposta da un "fare" che impedisce di "agire" e pensare il futuro con piena consapevolezza di sé". 
Il territorio non può essere, dunque, considerato come un mero contenitore.

Ove, infatti, vi siano determinate condizioni di armonia e consonanza nell'azione dei vari soggetti che insistono nella ricerca della soddisfazione delle varie istanze, esso può assurgere al ruolo di agevolatore/coordinatore delle risorse e delle attività economiche in esso presenti, il tutto in un'ottica di armonizzazione con le tradizioni culturali e naturali del luogo stesso.

L'impresa che adotta questa prospettiva considera il suo sviluppo economico come un obiettivo che trova piena realizzazione solo allorquando si coniuga con altre finalità che attengono al territorio su cui insiste, e che possono riconoscersi: nella qualità della vita, nella conservazione delle bellezze paesaggistiche, nell'ottimizzazione delle risorse disponibili, quindi nella crescita sociale: si pensi in proposito alla fondamentale esperienza della Olivetti di Adriano degli anni Cinquanta.

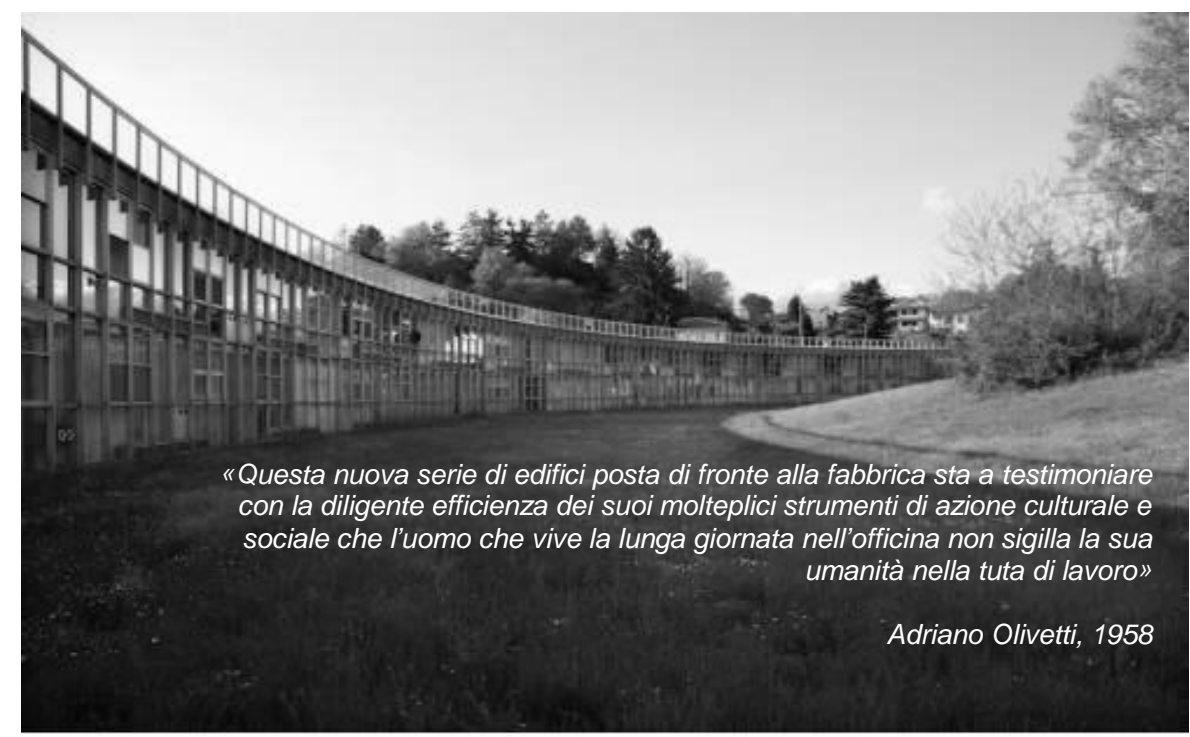

Fonte: Fondazione Adriano Olivetti, Centro Residenziale Ovest, 1968-1971, Architetti: Roberto Gabetti, Almaro Isola

Questa condizione è assolutamente in linea con l'imperativo dell'efficienza poiché: lo sviluppo socio-economico di un territorio funge da acceleratore nei processi di crescita dell'impresa, attraverso una logica di interscambio biunivoco. Tale asserzione, oltre ad essere confermata dall'esperienza distrettuale italiana, trova particolare dimostrazione nell'economia della conoscenza, ove si osservino casi come la Silicon Valley. 


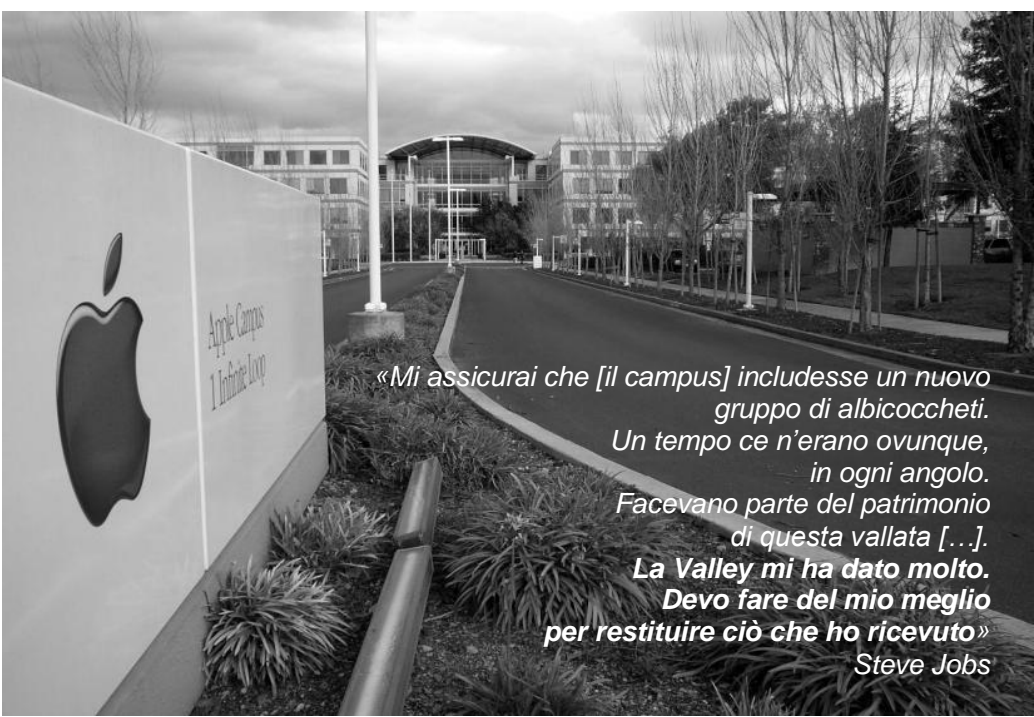

Fonte: http://www.flickr.com/photos/raneko/2373190256/

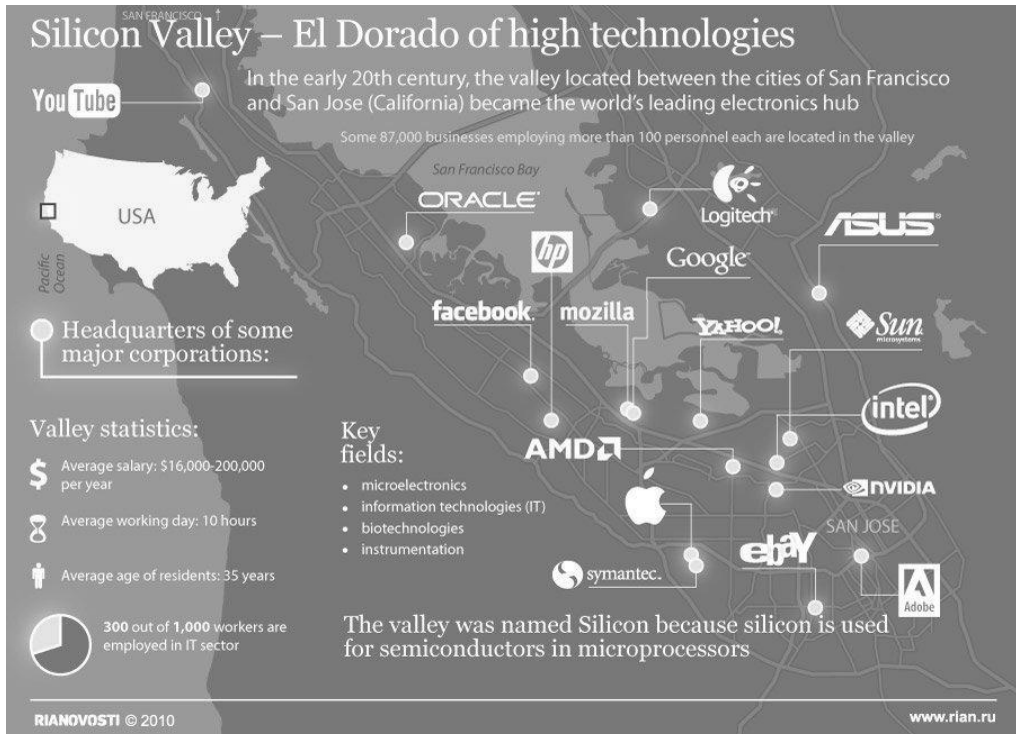

Fonte: http://en.rian.ru

Ieri il benessere del territorio dipendeva dalla presenza delle imprese. Oggi il benessere dell'impresa dipende anche dai caratteri del territorio. La competitività non è più solo tra singole imprese ma tra territori. 
Il territorio è così il luogo in cui l'impresa trova le ragioni del suo essere e del suo divenire, attingendo al giacimento del "capitale sociale" disponibile nelle forme introdotte da James Coleman (1990).

Quando ciò accade, si generano economie locali che possono trovare il vantaggio competitivo nell'interdipendenza e complementarietà delle loro formule; quindi non necessariamente nella gerarchia o nella subordinazione. Tale modalità di sviluppo non sempre nasce spontaneamente (quindi in maniera endogena), talvolta necessita di stimoli e di coordinamento che possono provenire dalle Istituzioni locali, le quali dovrebbero individuare le coerenti traiettorie di crescita dei territori considerandone le vocazioni e le potenzialità, in modo da raggiungere l'equilibrio, proprietà che caratterizza le entità sistemiche (Maturana e Varela, 1985).

Un risultato perseguibile con detta impostazione può essere il raggiungimento di condizioni del rapporto "territorio - prodotto" tali da determinare la tipicità/unicità dell'offerta. Il perseguimento di questo obiettivo (assimilabile al concetto di diversità intesa, in ambito agronomico, come effetto delle peculiarità del terroir) rende le produzioni di un territorio competitive, poiché non replicabili. Ciò, quindi, consente di porsi in una condizione antitetica all'omologazione, ovvero all'esasperata standardizzazione.

Perché tale circostanza giunga ad avere un valore economico-sociale particolarmente rilevante, è necessario che l'unicità si coniughi con la notorietà propria delle politiche di branding -, elevando così il territorio ad elemento fondamentale per l'attrattività dei luoghi e dei suoi contenuti.

Il territorio può così diventare un baluardo posto a difesa delle specificità produttive, delle tradizioni, della cultura dei luoghi, proponendo un'offerta differente dalle logiche dettate dalla globalizzazione spinta. Si può osservare, in proposito, come in risposta a tale ineludibile fenomeno, sia oggi cresciuta una sensibilità verso la preservazione del "locale", inteso come l'insieme dell'offerta armonica che un territorio riesce a proporre in termini di sintesi dei suoi prodotti non soltanto tangibili ma anche e soprattutto intangibili.

La ricerca di equilibrio della predetta contrapposizione (globale vs locale) può considerarsi un'interpretazione del termine glocalizzazione con cui Zygmunt Bauman (2005) enfatizza il ruolo delle comunità locali, cercando di porle in maniera antitetica e complementare rispetto ai comportamenti posti in essere dai modelli di omogeneizzazione. S'intende così sottolineare l'importanza del rispetto del territorio, delle sue tradizioni e culture, in modo da limitare gli effetti dell'eccessiva rilevanza socio-economica della globalizzazione. Il presupposto di partenza di tale approccio è rappresentato dal concepire preliminarmente il ruolo e le esigenze dei "sistemi più semplici" (quindi dei contesti territoriali), per poi giungere ad affrontare quelli più complessi, adottando il "principio della ricorsività" sistemica (Beer, 1991).

In questa maniera, si potrebbe evitare di ignorare gli effetti negativi che un incontrollato sviluppo economico può generare sui territori su cui si insedia.

Non si vuole con ciò contrastare od omettere di considerare l'importanza della globalizzazione, ma si ritiene necessario evidenziare il bisogno di riposizionare 
correttamente il ruolo del "locale" nel nuovo contesto; da ciò il motto "think global, act local" con cui si trasmette l'importanza di una corretta sintesi tra pensiero globale ed approccio locale.

Secondo tale impostazione, dunque, la predetta contrapposizione viene smussata e può quindi attenuarsi, poiché il locale rappresenta una modalità di offerta differente, alternativa e peculiare rispetto alla, non necessariamente negativa, offerta globale.

Va comunque rammentato che, come in molti fenomeni, anche il localismo non deve estremizzarsi. Non è, infatti, possibile o comunque utile pensare di enfatizzare oltremodo i singoli siti e le località poiché si perverrebbe ad una frammentazione talmente elevata dell'offerta da rendere vano qualsiasi sforzo di marketing e di comunicazione. Queste ultime azioni, infatti, necessitano di particolare cura ed attenzione sia nel momento della loro definizione strategica, sia in fase di attuazione. Il valore del territorio in quanto tale, infatti, come già detto, lo si raggiunge quando esso, oltre ad essere peculiare, diventa noto.

In proposito, è bene osservare come l'eccessiva proliferazione di azioni di marketing territoriale e/o di branding locale talvolta appaiano sterili, quindi, non efficaci, poiché generano confusione nei riceventi, vanificando così gli sforzi che talune attività congiunte potrebbero raggiungere più agevolmente in presenza di un coordinamento adeguato e con l'adozione della logica sistemica.

Ciò implica che:

Non tutti i territori hanno i caratteri della peculiarità.

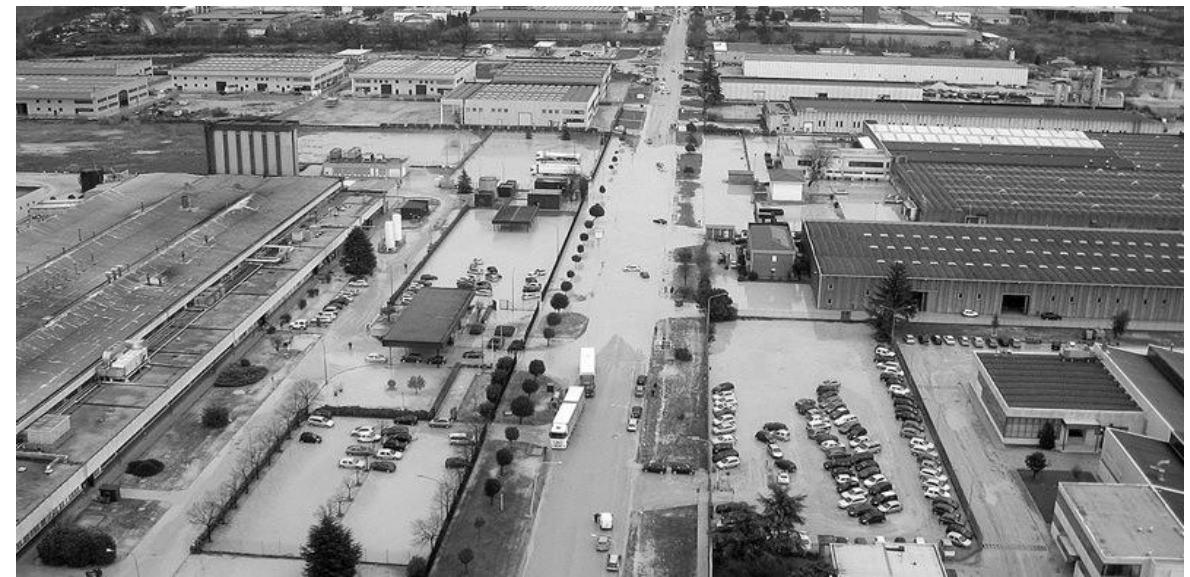

Solo alcuni territori peculiari riescono a raggiungere la notorietà. 


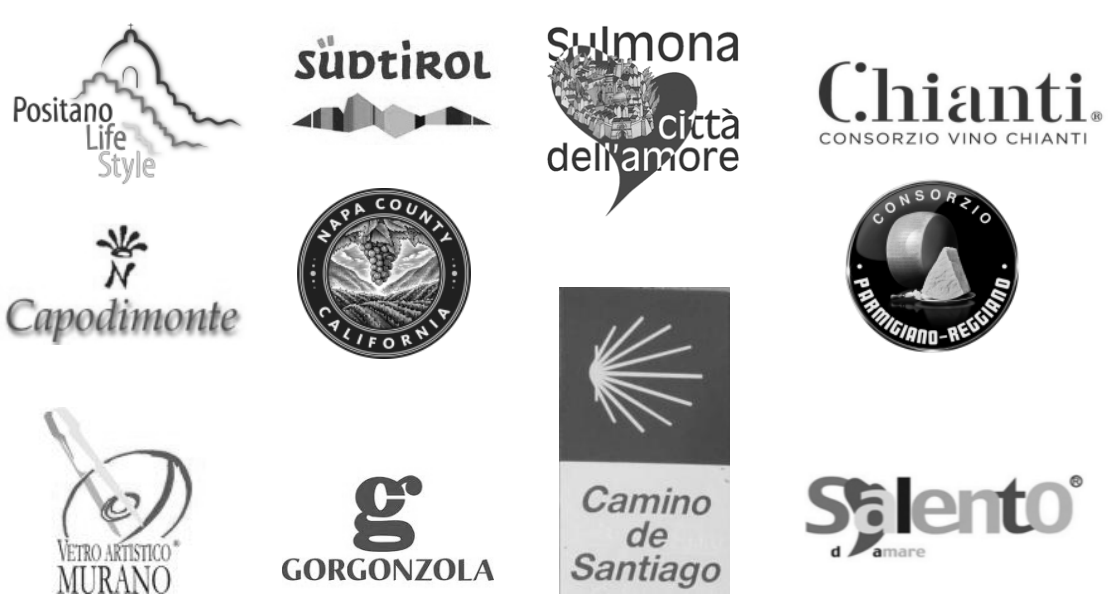

Questa evidenza non è sempre compresa: il limite del localismo è, infatti, il "pensiero locale".

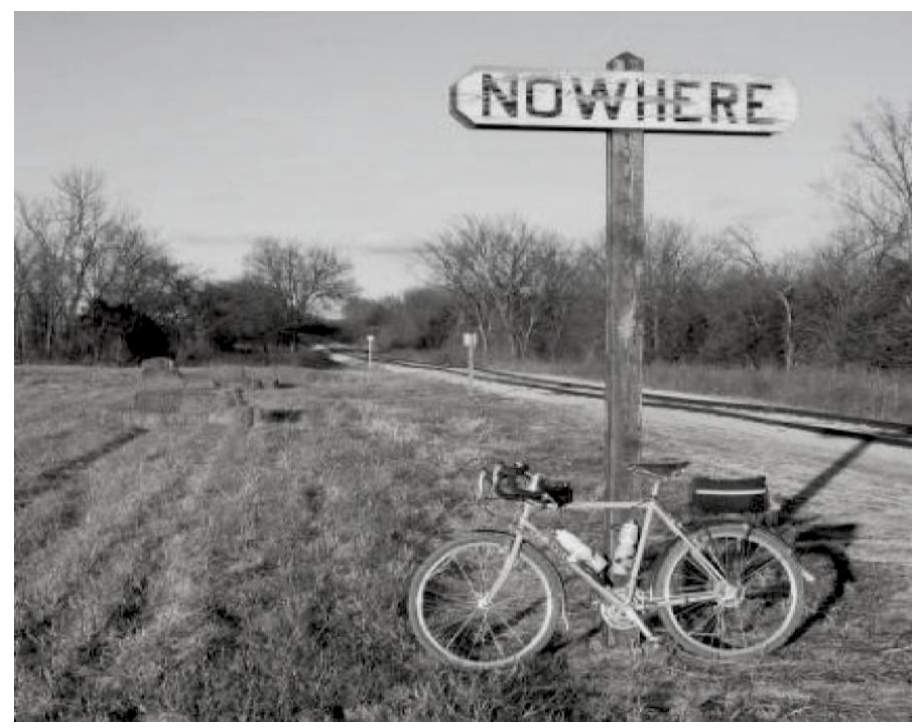

Non è, infatti, possibile pensare di poter affrontare oggi il mercato (appunto globale) con un'offerta particolarmente limitata quantitativamente o poco fruibile per via di un'eccessiva peculiarità o scarsità dell'assortimento.

È dunque necessaria un'azione di screening utile a discernere le tipologie produttive su cui puntare e i mercati verso cui rivolgersi. Per fare ciò, è necessario un coordinamento posto in essere da governance adeguate e capaci di sintetizzare le diverse specificità territoriali. 
Le potenzialità, insomma, delle formule sistemiche locali devono, ovviamente, riconoscersi nella sintesi delle varie caratteristiche territoriali, in modo da poter presentare un'offerta idonea rispetto alle attuali tendenze del mercato.

$\mathrm{Si}$ tratta, in definitiva, di riuscire a contenere il rischio del localismo, comprendendo che il valore del tutto discende anche dalla capacità delle parti di saper cogliere i propri limiti, adeguandosi alle più generali esigenze e prospettive strategiche.

Il tema è, dunque, assai ampio e investe problematiche di carattere trasversale a diversi ambiti scientifici nei quali, credo, il nostro può avere un giusto risalto. Gli studi di management, infatti, devono offrire il loro importante contributo per lo sviluppo socio-economico dei territori.

In tal senso, i numerosi paper giunti e articolati nelle diverse sessioni parallele ci fanno ben sperare.

\section{Bibliografia}

BACCARANI C., GOLINELLI G.M. (2011), "Per una rivisitazione delle relazioni tra impresa e territorio", Sinergie, n. 84, pp. VII-XIII.

BAUMAN Z. (2005), Globalizzazione e glocalizzazione, Armando editore, Roma.

BEER S. (1991), Diagnosi e progettazione organizzative, Isedi, Torino.

COLEMAN J.S. (1990), Foundations of social theory, Harvard University Press, Cambridge, Massachusetts.

LORENZONI G. (1990), L'architettura di sviluppo delle imprese minori. Costellazioni e piccoli gruppi, Il Mulino, Bologna.

MATURANA H., VARELA F. (1985), Autopoiesi e cognizione, Marsilio, Padova.

PANATI G. (1980), Politiche di ristrutturazione industriale e decentramento produttivo, Cedam, Padova.

RULLANI E. (1999), "L'impresa e il suo territorio: strategie di globalizzazione e di radicamento sul territorio", Sinergie, n. 49, pp. 25-31.

VARALDO R. (1995) "Dall'impresa localizzata all'impresa radicata", Economia Marche, n. 1, pp. 3-25. 
